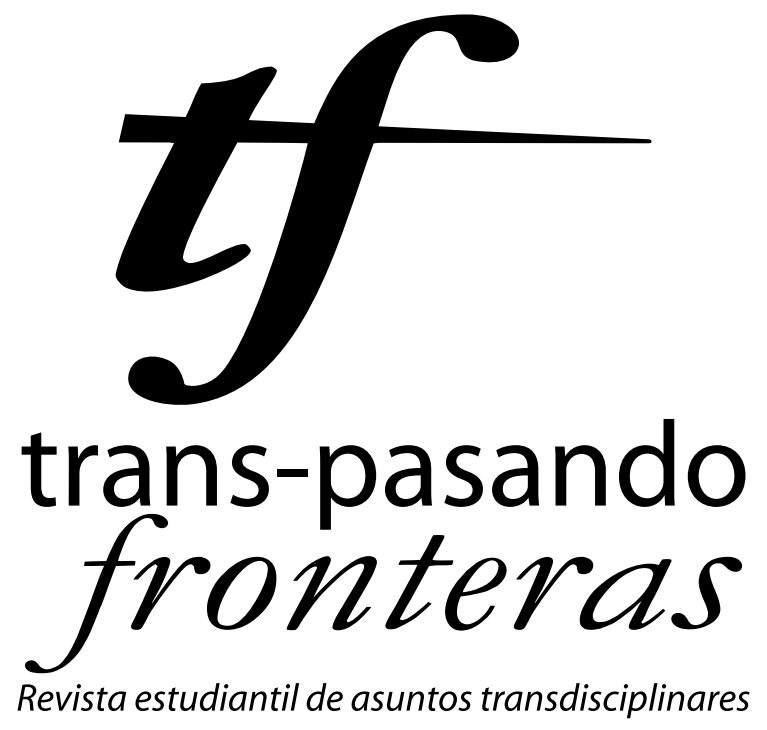

Una publicación de

Facultad de Derecho y Ciencias Sociales

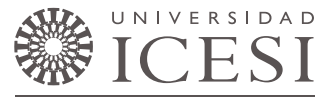




\title{
¿El principio del fin de las relaciones colombo-venezolanas?
}

\author{
Laura M. Pineda \\ (laurita0110@hotmail.com)
}

PASTRANA B., Eduardo, Carsten Wieland y Juan C. Vargas (2008). Vecindario agitado. Colombia y Venezuela: entre la hermandad y la conflictividad. Bogotá: Pontificia Universidad Javeriana, Facultad de Ciencias Políticas y Relaciones Internacionales. 272 páginas.

En los últimos años, las relaciones enInicialmente, es necesario que se estatre Colombia y Venezuela han sido fuente de noticias y discusiones. Han sido analizados los discursos del Presidente Chávez y de Uribe, ahora último, el de Santos también, presentando las diferentes facetas por las que han pasado las relaciones entre estos dos Estados, mutua y regionalmente catalogados como países "hermanos". A partir del análisis de cada capítulo por separado, se dará cuanta de cómo el libro Vecindario agitado. Colombia y Venezuela: entre la hermandad y la conflictividad y la teoría constructivista pueden ser útiles para estudiar cómo se perciben las relaciones internacionales de estos dos países. blezcan los parámetros de la teoría constructivista $^{1}$ de las relaciones internacionales. Históricamente, ésta aparece hacia los años 80 y permite "una visión más dinámica y menos rígida de las relaciones entre estructuras y actores" en las que se tiene en cuenta, por un lado, el contexto de cada relación y, por el otro lado, la manera en como estos actores se desenvuelven. Para el constructivismo, las relaciones internacionales no se mueven tras el poder

1 Para hablar sobre lo que constituye esta teoría, nos apoyaremos en lo que escribe Alexander Wendt sobre el constructivismo en La anarquía es lo que los estados hacen de ella. La construcción social de la política de poder, y artículos sobre el constructivismo. 
material como las fuerzas militares, las capacidades económicas, los balances de poder que condicionan el comportamiento de los actores, sino que aquí se privilegia una mirada en clave de lo social. Como lo dice Alexander Wendt, las teorías sociales "buscan explicar las identidades y los intereses" de los actores, mostrar cómo las instituciones pueden transformar las identidades y los intereses.

Uno de los principios del constructivismo es que "la gente se relaciona con los objetos, incluyendo otros actores, según el significado que estos tienen para ellos". Es importante tener en cuenta que, según Wendt, "los actores adquieren identidad mediante su participación en los significados colectivos" que son los que "conforman las estructuras que organizan nuestras acciones". De este modo, lo que trataremos de hacer, a partir de este libro, es determinar cómo se han visto afectadas las relaciones entre Colombia y Venezuela, a partir del cambio de los intereses de cada uno y las identidades.

El primer capítulo, Colombia y Venezuela en tres momentos: del panamericanismo al latinoamericanismo, nos deja ver cómo estos países detentan un origen común a partir de la idea de la Gran Colombia de Simón Bolívar. En ese entonces, el punto en cuestión trataba de países en una lucha contra la dominación de la corona española, sus identidades convergían.

Del mismo modo, hablar una misma lengua y compartir fronteras en común, permite que surja cierto deseo de "panamericanismo", idea que no va a ser apoyada por los Estados Unidos por su falta de identificación con el mismo. Luego, comienza a emerger la idea de "latinoamericanismo" como alternativa. Éste busca posicionarse como una fuerza regional a partir de las relaciones entre países sudamericanos con acuerdos sobre todo comerciales, pues sus intereses son, en muchos casos, comunes. Se trata de la búsqueda de una identidad latinoamericana fuerte a nivel mundial.

\section{Colombia-Venezuela: la hiperinfla-} ción del ejecutivo, compara las dictaduras latinoamericanas con la concentración de poder por parte del presidente en Colombia y Venezuela. Esta centralización del poder se da por el creciente miedo a una cierta disminución a causa de los conflictos de tipo social, como las guerrillas y los movimientos socialistas.

Lo que decidieron los mandatarios fue encontrar respaldo haciéndose jefes de las fuerzas armadas los cuales, a partir de allí, toman un valor importante en cuanto 
a las relaciones internacionales, tan importante que por ejemplo el gobierno de Chávez le apuesta a tener armamento que lo proteja contra cualquier posible ataque de Estados Unidos, claro ejemplo de dos países con intereses encontrados; lo anterior le conviene a Colombia en tanto su identidad siga manchada por la existencia de grupos armados ilegalmente y que uno de sus principales intereses sea la pretensión de eliminarlos.

En esta misma línea sigue el capitulo que trata de las relaciones civiles militares en Colombia y Venezuela: un reto para la democracia, ya que para los mandatarios, los militares siguen estando presentes en sus "proyectos políticos". Mientras en el caso Venezolano, la fuerza militar ha sido más fuerte que los partidos en la política, en Colombia los partidos son predominantes, sin embargo, los presidentes se han dado cuenta que para llevar a cabo sus intereses, es necesario contar con el apoyo de las fuerzas militares. Así vemos la forma como llega el militar Hugo Chávez al poder y por consiguiente, como se la han dado prerrogativas a los militares llegando a presenciarse fuertemente en otras instancias, sobre todo en el nivel burocrático administrativo, muy diferentes a lo relacionado al ejercito.
En Colombia, los militares se han visto involucrados en procesos de paz con las FARC, como es el caso de la desmilitarización en tiempos del despeje en el gobierno de Pastrana, al igual que en el gobierno de Uribe que por su política de seguridad democrática, logró legitimar recursos para el ejército, lo que incentivó su crecimiento y especialización.

En el capitulo de Colombia-Venezuela: dos maneras de comprender la seguridad, los autores plantea que esta "manera de comprender la seguridad es la divergencia que sostienen los dos países en cuanto a las relaciones con Estados Unidos". En este caso, es la percepción que cada uno tiene sobre Estados Unidos lo que determina sus identidades. Colombia, en su afán por contrarrestar los problemas con grupos al margen de la ley, que en un principio eran internos pero que se han convertido en problemas fronterizos con países como Ecuador, ve en Estados Unidos un aliado en este aspecto.

En cuanto a Venezuela y Ecuador, su soberanía se ha visto cuestionada. Hay aquí un conflicto entre "la seguridad del Estado y la seguridad humana". En el caso de Venezuela, por motivo de que su concepto de seguridad va muy ligado con el de "territorialidad", Estados Unidos representa una amenaza a ese mismo principio. 
En el quinto capítulo establece algunos puntos como diferencias ideológicas y aumento del narcotráfico: la posición ambivalente de Venezuela. Aquí hay un acercamiento a la identidad colombiana a partir de enunciados que tildan a Colombia como "primer productor a nivel mundial de sustancias psicotrópicas". Este es un gran peso con el que carga el gobierno Colombiano a diario, pues es una desacreditación internacional que ha dificultado establecer relaciones exteriores. Es aquí cuando entra otro tema importante: la ideología. Las guerrillas colombianas, que en principio actuaban bajo líneas comunistas pero se financian del narcotráfico. De lo anterior se arguye sobre si es necesario el apoyo de Estados Unidos ya que le conviene el cese de la producción de droga (ya que él mismo constituye el mayor consumidor), sino que permanece presente en el continente sudamericano.

Venezuela, claramente está en contra de esta presencia pues el Estado de Chávez se inclina hacia formas socialistas de gobierno y ven en Estados Unidos un "imperio capitalista". En este sentido, las identidades de ambos países se oponen, lo desencadena gran parte de sus desacuerdos.

En este próximo capítulo, Interdependencia comercial entre Colombia y Vene- zuela: tensiones y transformaciones con Uribe-Chávez, los autores presentan el enfrentamiento de las identidades con los intereses. Existe la tendencia en América Latina hacia la unión comercial para promover lazos económicos entre los países latinoamericanos, lo que se refleja en la creación de alianzas como MERCOSUR, UNASUR o la Comunidad Andina de Naciones. En lo concerniente a las relaciones comerciales entre Colombia y Venezuela es evidente que estos lazos están desde hace ya varias décadas fuertemente ligados. Para ambos países son importantes las importaciones provenientes del otro país, lo que permite descifrar que comparten este interés económico. Sin embargo, en la medida en que Venezuela se convierte cada vez más en una potencia petrolera de gran importancia a nivel mundial y pretende remplazar las relaciones comerciales que tiene con Colombia por la de otros países latinoamericanos, especialmente con aquellos que muestran más afinidad a su tipo de gobierno, queda en manifiesto como los rasgos identitarios de un país pueden cambiar, y en la medida en que van cambiando, la percepción de identidad por parte de los demás países, cambian también. Un ejemplo de lo anterior, es el deseo de los gobiernos de Uribe y Santos de crear 
lazos comerciales más fuertes con Estados Unidos implantando el Tratado de Libre Comercia - TLC, lo que ha sido fuente de discordia entre ambos países pero que en gobiernos anteriores a Chávez hubiese sido bien recibido.

El liderazgo populista de Chávez, su discurso y las relaciones colombo-venezolanas es un capitulo particular. Aquí los autores hablan de los mandatarios: Álvaro Uribe Vélez y Hugo Chávez Frías, específicamente sobre la forma en como cada presidente crea su identidad como presidente. Ambos llegan al poder sin el respaldo de un partido político especifico y es gracias a su nivel de carisma como lo logran. Los autores hablan en este capítulo de "populismo" y sin embargo, sus discursos son muy diferentes. Por un lado, Chávez tiende a una extra-exposición de sus opiniones buscando legitimar su proyecto de "revolución bolivariana" y de expandirla a otras naciones simpatizantes como Ecuador, Bolivia y Nicaragua. Por el otro lado, Uribe critica abiertamente la postura política del presidente Chávez y presenta cuanta evidencia sea necesaria de los nexos entre las FARC y el gobierno de Venezuela. Postura con la cual cuenta con los Estados Unidos, un fuerte aliado con el cual el bolivarianismo Chavista no está de acuerdo.
Finalmente, el ultimo capítulo, ¿Irreversibilidad de la degradación de las relaciones político- diplomáticas?, los autores plantean diferentes focos de tensión que pueden significar un divorcio en los intereses de Colombia y Venezuela. Aquí hablan de sus posturas opuestas de los "modelos de integración regional, de las relaciones con Estados Unidos, de la cooperación para luchar contra el terrorismo internacional", entre otras.

Estos son puntos álgidos en cuanto a las relaciones colombo- venezolanas y evidencia intereses no compartidos. Es frente a esto que giran y se construyen los diferentes elementos identitarios de cada país. La gran pregunta final es, de acuerdo con la sobre la identidad percevida por cada uno de estos actores, ¿podemos hablar de una irreversibilidad en las relaciones colombo-venezolanas? Mucho se ha hablado del rompimiento de las relaciones entre Colombia y Venezuela, sin embargo, estos libros no hacen, por lo general, un recorrido histórico de esta relación, sino que se centran en un periodo de corto plazo para abordar el porqué o por qué no podemos hablar del rompimiento de relaciones bilaterales de estos dos países. A partir de la teoría constructivista, vemos como esta relación sí está marcada por conflictos de 
intereses pero sobre todo, por la creación de formas de identidades que permiten entender, desde diferentes puntos de vista, el comportamiento de la relación entre Colombia y Venezuela. 